\title{
What Mass Are the Smallest Protohalos?
}

\author{
Stefano Profumo, ${ }^{1, *}$ Kris Sigurdson, ${ }^{2, \dagger}$ and Marc Kamionkowski ${ }^{1, *}$ \\ ${ }^{1}$ California Institute of Technology, Mail Code 130-33, Pasadena, California 91125, USA \\ ${ }^{2}$ Institute for Advanced Study, Einstein Drive, Princeton, New Jersey 08540, USA \\ (Received 15 March 2006; revised manuscript received 8 May 2006; published 18 July 2006)
}

\begin{abstract}
We calculate the kinetic-decoupling temperature for weakly interacting massive particles (WIMPs) in supersymmetric (SUSY) and extra dimensional models that can account for the cold-dark-matter abundance determined from cosmic microwave background measurements. Depending on the parameters of the particle-physics model, a wide variety of decoupling temperatures is possible, ranging from several $\mathrm{MeV}$ to a few $\mathrm{GeV}$. These decoupling temperatures imply a range of masses for the smallest protohalos much larger than previously thought-ranging from $10^{-6} M_{\oplus}$ to $10^{2} M_{\oplus}$. We expect the range of protohalos masses derived here to be characteristic of most particle-physics models that can thermally accommodate the required relic abundance of WIMP dark matter, even beyond SUSY and extra dimensions.
\end{abstract}

DOI: 10.1103/PhysRevLett.97.031301

The physical nature of dark matter remains one of the major unsolved problems in theoretical physics and cosmology. One of the leading candidates for dark matter is a weakly interacting massive particle (WIMP) [1]. In the simplest models, WIMPs (which we denote by $\psi$ ) carry a conserved quantum number that renders them stable. When the temperature in the early Universe drops below $m_{\psi}$, the WIMP abundance tracks the thermal equilibrium distribution and is Boltzmann suppressed until pairs of WIMPs can no longer find each other within a Hubble time, and the comoving number density of WIMPs becomes constant. Up to factors of a few, this freeze-out of the annihilation channel happens at a temperature $T_{\mathrm{fo}} \sim m_{\psi} / 20$ and leads to a relic abundance of dark matter of $\Omega_{\psi} h^{2} \simeq(3 \times$ $\left.10^{-27} \mathrm{~cm}^{3} \mathrm{~s}^{-1}\right) /\left\langle\sigma_{a} v\right\rangle$, where $\left\langle\sigma_{a} v\right\rangle$ is the thermally averaged cross section (times relative velocity) for annihilation of $\psi$ pairs into lighter particles. In typical models, $m_{\psi} \sim$ $100-1000 \mathrm{GeV}$, and $T_{\mathrm{fo}} \sim 5-50 \mathrm{GeV}$.

While freeze-out signals the departure of WIMPs from chemical equilibrium, it does not signal the end of WIMP interactions. Elastic and inelastic scattering processes of the form $\psi f \rightarrow \psi f$ or $\psi f \rightarrow \psi^{\prime} f^{\prime}$ keep the dark matter in kinetic equilibrium until later times (lower temperatures) [2-4]. Here $f$ and $f^{\prime}$ are standard model particles in the thermal bath (leptons, quarks, gauge bosons) and $\psi^{\prime}$ is an unstable particle that carries the same conserved quantum number as $\psi$. The temperature $T_{\mathrm{kd}}$ of kinetic decoupling sets the distance scale at which linear density perturbations in the dark-matter distribution get washed out - the smallscale cutoff in the matter power spectrum. In turn, this small-scale cutoff sets the mass $M_{c}$ of the smallest protohalos that form when these very small scales go nonlinear at a redshift $z \sim 70$. There may be implications of this small-scale cutoff for direct detection (detection of WIMPs scattering off ordinary matter in low-background environments) [5] and indirect detection (detection of the byproducts of WIMP-pair annihilations including antimatter, gamma rays, and neutrinos) [6].
PACS numbers: 98.80.Cq, 11.10.Kk, 12.60.Jv, 95.35.+d

Some early work assumed that the cross sections for WIMPs to scatter from light particles (e.g., photons and neutrinos) would be energy independent, leading to suppression of power out to fairly large (e.g., galactic) scales. However, in supersymmetric (SUSY) models, at least, the relevant elastic-scattering cross sections drop precipitously with temperature, resulting in much higher $T_{\mathrm{kd}}$ and much smaller suppression scales [3]. If the annihilation cross section of WIMPs into light fermions goes as $\sigma_{a} \simeq$ $g_{a}^{4} / m_{\psi}^{2}$, then one expects the scattering cross section to be $\sigma_{s} \simeq g_{s}^{4} E^{2} / m_{\psi}^{4}$, where $E$ is the energy of the scattering light particles, and $g_{a} \sim g_{s}$ up to factors of order unity. This estimate has been used to derive $T_{\mathrm{kd}}$ [7] and infer that the typical order of magnitude of the minimum protohalo mass is $M_{c} \sim M_{\oplus}[4,5,8]$. However, to date, no detailed calculation of $T_{\mathrm{kd}}$ and $M_{c}$ in supersymmetric or other models consistent with experimental and cosmological data has been performed.

In this Letter, we calculate the kinetic-decoupling temperature $T_{\mathrm{kd}}$ of WIMP dark matter in models that account for the correct cold-dark-matter density while remaining consistent with laboratory constraints. We consider models within the minimal supersymmetric extension of the standard model (MSSM), models with universal extra dimensions (UED), and also other extra dimensional models. Instead of relying on heuristic arguments or toy models, we use the detailed scattering cross sections of WIMPs, including resonances and threshold effects, both for the WIMP relic abundances and for $T_{\mathrm{kd}}$. The main result of our analysis is that $T_{\mathrm{kd}}$ may range all the way from tens of $\mathrm{MeV}$ to several GeV. These $T_{\mathrm{kd}}$ imply a range $M_{c} \sim 10^{-6} M_{\oplus}$ to $M_{c} \sim 10^{2} M_{\oplus}$, where we use the estimate [8]

$$
M_{c} \simeq 30\left(T_{\mathrm{kd}} / 10 \mathrm{MeV}\right)^{-3} M_{\oplus},
$$

which accounts for both the acoustic oscillations imprinted on the power spectrum by the coupling between the dark matter and the relativistic particles in the primordial 
plasma prior to kinetic decoupling and the cutoff due to free streaming of dark matter after kinetic decoupling. Although we focus on particular WIMP scenarios, we expect the range of $M_{c}$ found here will be characteristic of most particle-physics models that accommodate the required relic abundance of thermal WIMP dark matter.

We define $T_{\mathrm{kd}}$ from $\tau_{r}\left(T_{\mathrm{kd}}\right)=H^{-1}\left(T_{\mathrm{kd}}\right)$ [4], where $H(T)$ is the Hubble expansion rate, and the relaxation time $\tau_{r}$ is

$$
\tau_{r}^{-1} \equiv \sum_{l} n_{l}\left(T, m_{l}\right) \sigma_{l \psi}(T)\left(T / m_{\psi}\right)
$$

Here, $n_{l}\left(T, m_{l}\right) \sim T^{3}$ is the equilibrium number density of the relativistic particle species $l$ (the mass dependence is crucial here for the heavier species), $\sigma_{l \psi}(T)$ is the thermally averaged scattering cross section of the WIMP $\psi$ off $l$ 's, and the factor $\left(m_{\psi} / T\right)^{-1}$ counts the number of scatters needed to keep the WIMPs in kinetic equilibrium. Here, we consider $l \in\left\{\nu_{e, \mu, \tau}, e, \mu, \tau, u, d, s, c\right\}$ and neglect the scattering off quarks below $\Lambda_{\mathrm{QCD}} \simeq 150 \mathrm{MeV}$ (our results are insensitive to the precise value taken for $\Lambda_{\mathrm{QCD}}$ and the detailed nature of the QCD phase transition). We neglect the scattering of WIMPs off mesons and baryons below $\Lambda_{\mathrm{QCD}}$ because this process is suppressed with respect to their scattering off light leptons by the relative abundance of the species in the thermal bath and by hadronic form factors.

In the case of supersymmetric models, the scattering of neutralinos ( $\chi$ 's) off light fermions proceeds through sfermion and gauge-boson exchange. The relevant cross sections have been computed in Ref. [3] for neutralinoneutrino scattering. We extend here the results of Ref. [3] to include charged-lepton and quark scattering, where further diagrams (involving both right- and left-handed sfermion exchange) as well as novel interfering amplitudes appear. The scattering cross section $\sigma_{\chi l}$ goes as $E_{l}^{2}$ [3], modulo resonant channels where the exchanged sfermion mass is quasidegenerate with the neutralino mass. In this latter case, the sfermion width has to properly be taken into account in the computation of $\sigma_{\chi l}(T)$.

We show the neutralino-electron scattering cross section as a function of energy in Fig. 1, where we pick supersymmetric "benchmark" models in the context of minimal supergravity (mSUGRA) [9]. We set for all models (with the usual notation) $m_{1 / 2}=500 \mathrm{GeV}, A_{0}=0, \tan \beta=10$, $\mu>0$, and $m_{t}=172.7 \mathrm{GeV}$; in all cases, the neutralino mass is around $200 \mathrm{GeV}$. Model A features $m_{0}=$ $100 \mathrm{GeV}$, and lies in the coannihilation region, where scalar superparticles are light, and the next-to-lightest supersymmetric particle (NLSP) is a $\tau$ slepton. The latter is, here, quasidegenerate with the lightest neutralino, and coannihilation brings the neutralino relic abundance into accord with the dark-matter abundance. Model B belongs, instead, to the focus-point region, where large scalar masses (here, $m_{0}=2770 \mathrm{GeV}$ ) at the grand-unification scale drive the Higgsino mass parameter $\mu$ to low values at the weak scale through renormalization-group evolution

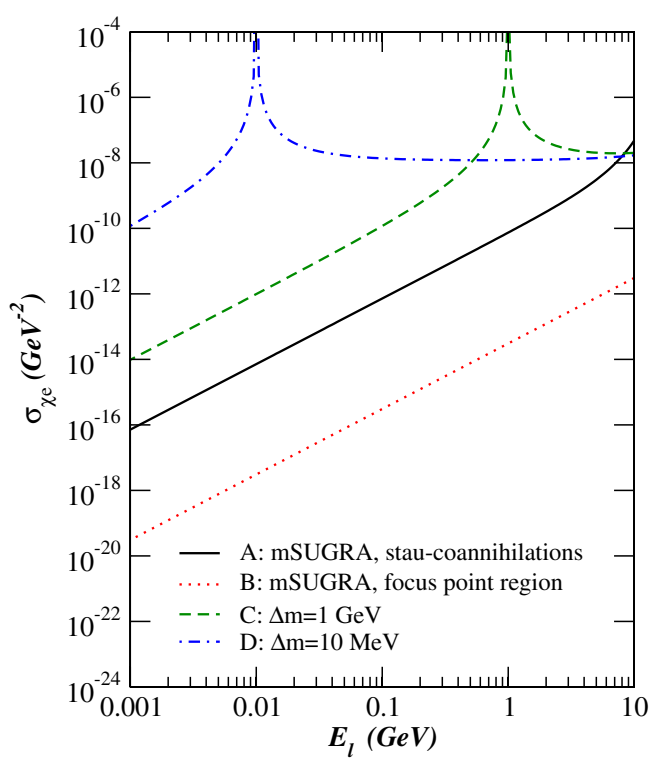

FIG. 1 (color online). Neutralino-electron scattering cross section as a function of the electron energy $E_{l}$ for the four benchmark models A-D discussed in the text.

and radiative electroweak-symmetry breaking. A low value of $\mu$ implies a mixed higgsino- $B$-ino dark-matter particle. Heavy sfermions imply that scattering off light fermions proceeds through $Z^{0}$ exchange, and the resulting $\sigma_{\chi l}$ is suppressed with respect to the light-sfermion case (model A) by almost 4 orders of magnitude.

We also examine models that exhibit the effects of sfermion resonances in neutralino-fermion scattering. We modify model A, lowering the soft-supersymmetrybreaking left-handed slepton masses of the first two generations, in order to get $\Delta m_{\tilde{\nu}_{e, \mu}} \equiv m_{\tilde{\nu}_{e, \mu}}-m_{\chi} \simeq \Delta m_{\tilde{e}_{1}, \tilde{\mu}_{1}}=$ $1 \mathrm{GeV}$ (model C) and $0.01 \mathrm{GeV}$ (model D). These models can be motivated in the context of extensions of mSUGRA with nonuniversal scalar masses [see, e.g., Ref. [10]]. At sufficiently small temperatures, $\sigma_{\chi l} \propto E_{l}^{2}$ is recovered, but for $T \gtrsim \Delta m_{\tilde{\nu}}, \sigma_{\chi l} \simeq$ constant, and is simply set by the neutralino mass and by the relevant neutralino-leptonslepton couplings $\left(\sigma_{\chi l} \propto\left|g_{\chi}\right|^{4} / m_{\chi}^{2}\right)$.

Another class of WIMP models that has recently received considerable attention is that arising in the context of universal extra dimensions (UED) [11]. A viable lightest Kaluza-Klein particle (LKP) dark-matter candidate is the first Kaluza-Klein (KK) excitation of the $U(1)$ gauge boson $B^{(1)}$. Precision electroweak measurements [12], the LKP relic abundance [13], and direct-detection experiments [14] strongly constrain the viable range of masses for the LKP. However, this range depends sensitively upon the details of the spectrum of the first and second KK excitations, which can include significant coannihilation [15] and resonant-annihilation effects. We compute here the scattering cross section of $B^{(1)}$ off light fermions, for which the relevant Feynman diagrams are shown in Fig. 2(a). We expect large scattering cross sections in this case, since the 


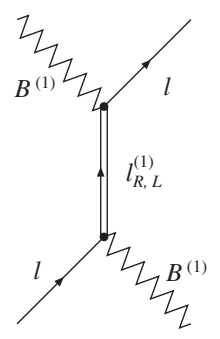

(a.1)



(a.2)

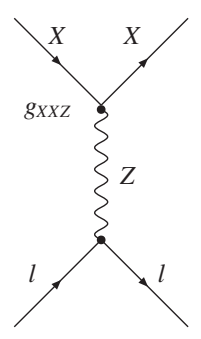

(b)
FIG. 2. Feynman diagrams contributing to the scattering of a $B^{(1)}$ LKP (a.1 and a.2) and a $\nu$-like $X$ particle (b) off fermions.

intrinsically degenerate nature of the KK spectrum, where $m_{B^{(1)}} \simeq m_{l^{(1)}}$, clearly enforces a resonant enhancement. We find, to leading order in $E_{l} / m_{B^{(1)}}$, and in the relativistic limit for $l$ and nonrelativistic limit for the LKP particle, that

$$
\sigma_{B^{(1)} l} \simeq \frac{E_{l}^{2}}{2 \pi} \sum_{R, L} \frac{\left(g_{1} Y_{R, L}\right)^{4}}{\left(m_{B^{(1)}}^{2}-m_{l_{R, L}^{(1)}}^{2}\right)^{2}},
$$

where $Y_{R, L}$ stand for the hypercharge quantum numbers. Finally, we consider an alternative class of models that feature a heavy neutrinolike dark-matter particle $X$ coupled to the $Z^{0}$ through a generic coupling $g_{X X Z}$, Fig. 2(b) [specific cases include, for instance, the Dirac right-handed neutrino of 5D warped grand-unification [16] or the KK neutrino $\nu^{(1)}$ of UED]. In this case

$$
\sigma_{X l} \simeq \frac{\left|g_{X X Z}\right|^{2}}{4 \pi m_{Z}^{4}}\left(g_{L}^{2}+g_{R}^{2}\right) E_{l}^{2},
$$

where $g_{R, L}$ stand for the $L$ and $R$ couplings of the fermion $l$ to the $Z^{0}$ gauge boson. Interestingly, $\sigma_{X l}$ does not depend on $m_{X}$. We note that the $\sigma_{\psi l} \propto E_{l}^{2}$ scaling found in the case of neutralino dark matter is valid for these two alternative classes of WIMPs as well.

Different WIMP models give rise to different $T_{\mathrm{kd}}$, and therefore to different $M_{c}$. We apply our elastic-scattering cross section for WIMPs from light fermions to the MSSM parameter space, following the scan procedure of Ref. [17], requiring that the neutralino density falls within the WMAP $2 \sigma$ range for the dark-matter density [18]. Figures 3 and 4 show our results for $T_{\mathrm{kd}}$ and for $M_{c}$, respectively, versus the WIMP mass. We also scan over a subset of the MSSM, defined by the mSUGRA setup: the results of this second scan are shown as light blue filled dots in the figures. We stress that although within mSUGRA special mechanisms (coannhilation, resonances, etc.) are generically required to achieve the correct neutralino relic abundance, we find nearly as broad a range of $T_{\mathrm{kd}}$ in this case as we do in the theoretically unrestricted MSSM (which for Higgsino or $W$-ino-like neutralinos does not generically require special relic density suppression mechanisms). The physical reason for the large range of $T_{\mathrm{kd}}$ compatible with thermal neutralino production depends on (i) the fact that chemical and kinetic decouplings involve different micro-



FIG. 3 (color online). The kinetic-decoupling temperature $T_{\mathrm{kd}}$ as a function of the WIMP mass for supersymmetric models (red empty dots are for the general MSSM while light-blue filled dots are for mSUGRA) giving a neutralino thermal relic abundance consistent with cosmology, for UED models featuring a $B^{(1)}$ LKP, and for a model featuring a $\nu$-like $X$ particle. The four benchmark models A-D discussed in the text are also shown.

physical processes, (ii) the wide range of sfermion masses considered, possibly giving rise to large resonant enhancements or large-mass suppressions in the WIMP-fermion scattering, and (iii) the wide spread of WIMP- $Z^{0}$ couplings

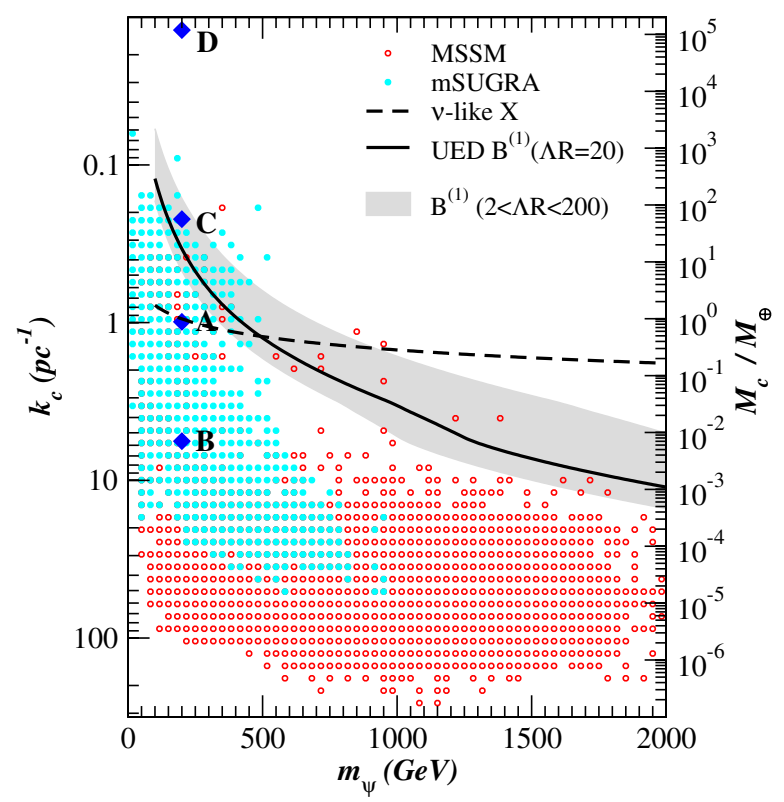

FIG. 4 (color online). The WIMP protohalo characteristic comoving wave number $k_{c}$ (left axis) and mass $M_{c}$ (right axis) as a function of the WIMP mass, for the same models as in Fig. 3. 
induced by gaugino-higgsino mixing, particularly relevant in the limit of heavy sfermions.

We indicate the range of results expected for a $B^{(1)} L K P$. We set the KK spectrum according to the minimal UED prescription for radiative corrections to the $\mathrm{KK}$ masses [19], setting the cutoff scale $2<\Lambda R<200$, and showing the "standard" $\Lambda R=20$ case with a black solid line. We further show the case of a heavy neutrinolike particle $X$ coupled to the $Z^{0}$, setting the coupling $g_{X X Z}=g_{\nu^{(1)} \nu^{(1)} Z}=$ $e / \sin 2 \theta_{W}$ for illustrative purposes. Finally, we also indicate the four benchmark models of Fig. 1 discussed above.

We conclude that (i) in most supersymmetric models, $5 \lesssim T_{\mathrm{kd}} / \mathrm{MeV} \lesssim 3000$, but even lower values can be attained with finely tuned resonant channels; (ii) the expected range of $M_{c}$ varies over the wide range $10^{-6} \lesssim M_{c} / M_{\oplus} \lesssim 10^{2}$ - a much wider range of masses than previously estimated [4]; (iii) $B^{(1)}$ LKPs typically decouple later than neutralinos (around $40 \mathrm{MeV}$ for values of $m_{B^{(1)}}$ preferred by the thermal relic abundance and by electroweak measurements); and (iv) combining Eqs. (2) and (4) we find that the general case of $X$ particles which scatter off light fermions through $Z^{0}$ exchange with a coupling $g_{X X Z}$ produces protohalos with mass

$$
M_{c} \approx 100 M_{\oplus}\left|g_{X X Z}\right|^{3 / 2}\left(m_{X} / 100 \mathrm{GeV}\right)^{-3 / 4} \text {. }
$$

Since the relevant quantities for WIMP-nucleon scattering and for the annihilation of WIMPs into gamma rays or antimatter are typically poorly correlated with WIMPfermion scattering, conventional dark-matter-detection rates cannot be simply related to $T_{\mathrm{kd}}$. However, we point out that a low $T_{\mathrm{kd}}$ implies large WIMP-lepton scattering cross sections and may produce sizable signals at future electron accelerators using the search technique recently proposed in Ref. [20]. If the masses of the sleptons and neutralino can be measured by future colliders then, if $\Delta m$ is small enough, the beam energy of a future electron accelerator might be tuned to $E_{\text {beam }} \simeq \Delta m$, enabling resonant $s$-channel scattering of neutralino dark matter. The scattered electrons could then be detected by calorimeters or tracking chambers along the beam line. This technique would also reveal information about the dark-matter velocity distribution [20]. As a rule of thumb, more than one event per year is expected at a future electron collider with $100 \mathrm{~m}$ of detector length and $10 \mathrm{~A}$ of beam current if $T_{\mathrm{kd}} \lesssim$ $10 \mathrm{MeV}$; in the extreme case of $T_{\mathrm{kd}} \approx 1 \mathrm{MeV}$, the expected event rate per year could be as large as $\sim 10^{4}$. If these types of WIMP-lepton recoil events are in fact detected we will have a laboratory estimate of the WIMP decoupling temperature.

The temperature $T_{\mathrm{kd}}$ in WIMP models has a critical impact not only on the size distribution of primordial protohalos expected in $\mathrm{N}$-body simulations of structure formation [5], but also for potential effects in WIMP direct and indirect detection induced by dark-matter clumps or streams, or in the anisotropy of the cosmic gamma-ray background induced by WIMP annihilations [6]. Accounting for the wide range of possibilities consistent with detailed WIMP models should therefore be regarded as an essential ingredient for future studies in this field.

K. S. is supported by NASA through Hubble Fellowship Grant No. HST-HF-01191.01-A awarded by the Space Telescope Science Institute, which is operated by the Association of Universities for Research in Astronomy, Inc., for NASA, under Contract No. NAS 5-26555. S. P. and M. K. are supported in part by DOE No. DE-FG03-92ER40701 and FG02-05ER41361 and NASA No. NNG05GF69G.

*Electronic address: profumo@caltech.edu

${ }^{\dagger}$ Electronic address: krs@ias.edu

Electronic address: kamion@tapir.caltech.edu

[1] G. Jungman et al., Phys. Rep. 267, 195 (1996); L. Bergstrom, Rep. Prog. Phys. 63, 793 (2000); G. Bertone et al., Phys. Rep. 405, 279 (2005).

[2] C. Bœhm et al., Phys. Lett. B 518, 8 (2001).

[3] X. L. Chen et al., Phys. Rev. D 64, 021302(R) (2001).

[4] S. Hofmann et al., Phys. Rev. D 64, 083507 (2001); A. M. Green et al., Mon. Not. R. Astron. Soc. 353, L23 (2004); J. Cosmol. Astropart. Phys. 08 (2005), 003.

[5] J. Diemand et al., Nature (London) 433, 389 (2005); Astrophys. J. (to be published).

[6] V. Berezinsky et al., Phys. Rev. D 68, 103003 (2003); 73, 063504 (2006); T. Oda et al. Astrophys. J. 633, L65 (2005); S. Ando and E. Komatsu, Phys. Rev. D 73, 023521 (2006).

[7] C. Schmid et al., Phys. Rev. D 59, 043517 (1999).

[8] A. Loeb and M. Zaldarriaga, Phys. Rev. D 71, 103520 (2005).

[9] A. H. Chamseddine et al., Phys. Rev. Lett. 49, 970 (1982).

[10] H. Baer et al., J. High Energy Phys. 06 (2004), 044; S. Profumo, Phys. Rev. D 68, 015006 (2003).

[11] T. Appelquist et al., Phys. Rev. D 64, 035002 (2001); G. Servant and T.M.P. Tait, Nucl. Phys. B 650, 391 (2003).

[12] T. Flacke et al., Phys. Rev. D 73, 095002 (2006).

[13] K. Kong and K. T. Matchev, J. High Energy Phys. 01 (2006), 038; F. Burnell and G. D. Kribs, Phys. Rev. D 73, 015001 (2006); M. Kakizaki et al., Nucl. Phys. B 735, 84 (2006).

[14] G. Servant and T. M. P. Tait, New J. Phys. 4, 99 (2002).

[15] S. Matsumoto and M. Senami, Phys. Lett. B 633, 671 (2006).

[16] K. Agashe and G. Servant, J. Cosmol. Astropart. Phys. 02 (2005), 002; D. Hooper and G. Servant, Astropart. Phys. 24, 231 (2005).

[17] S. Profumo and C.E. Yaguna, Phys. Rev. D 70, 095004 (2004).

[18] D. N. Spergel et al., Astrophys. J. Suppl. Ser. 148, 175 (2003).

[19] H. C. Cheng et al., Phys. Rev. D 66, 036005 (2002).

[20] J. Hisano et al., Phys. Rev. D 73, 031701 (2006). 